\title{
Morteros de albañilería con escombros de demolición
}

\author{
JORGE L. ÁLVAREZ CABRERA, Ingeniero. Centro Técnico para el Desarrollo de los Materiales de Construcción \\ FRANCISCO URRUTIA, Arquitecto. Centro Técnico para el Desarollo de los Materiales de Construcción \\ DEBORAH LECUSAY, Arquitecta. Inversiones. Corporación CIMEX, S. A. \\ Fecha de recepción: $27-$ II-97
}

Fecha de aceptación: 20-III-97

CUBA

\section{RESUMEN}

La posibilidad real del reciclaje de escombros, su efectividad técnica, económica, y el impacto social y ecológico que proporciona a la sociedad, es nuestro punto de partida para lograr un óptimo aprovechamiento de este árido como futuro agregado fundamental en la obtención de un mortero de albañilerí, capaz de tener como propiedades aquellas intrinsecas de los propios morteros obtenidos a partir de áridos de cantera y recogidos en la norma cubana NC52-79:93.

El trabajo consiste en la preparación y estudio de morteros de albañilería empleando árido reciclado -producto de demolición- como agregado. Se prepararon 4 dosificaciones volumétricas $(1: 4 ; 1: 5 ; 1: 6$ y 1:8), las cuales fueron sometidas a ensayos físico-mecánicos a 7,28 y 60 días, resistencia a la adherencia a 28 dias, y absorción capilar, obteniéndose resultados comparables con los morteros de albañilería tradicionales.

\section{SUMMARY}

The possibility of recycling construction rubble, its technical and economic effectiveness, and the social and ecological impact they provide for our society is the reason and the starting point for an optimal exploitation of this aggregate in the manufacture of masonry mortar, since it has the same inherent properties as those obtained from the quarry aggregates, described in the cuban standard NC.52-79:93.

The work consists in the preparation and study of masonry mortars using recycled aggregates -demolition productFour volumetric dosages were prepared $(1: 4 ; 1: 5 ; 1: 6$ y $1: 8)$, which were submitted to physical and mechanical tests at 7,28 and 60 days, adherence resistance at 28 days, and capillar absorption, obtaining similar results to those

\section{INTRODUCCIÓN}

La escasez, creciente cada día, de materiales para la construcción y reparación de viviendas ha hecho que se piense en soluciones alternativas, como son el rescate -en lugares donde existen demoliciones o se producen derrumbes- de materiales como ladrillos, vigas, maderas y finos residuales. Es, a partir de estos últimos, donde surge la idea de su utilización y aprovechamiento, a mayor escala, para su empleo en morteros, así como por la posibilidad real del reciclaje de dichos escombros, su efectividad técnica y económica y el impacto social y ecológico que proporciona a la sociedad, para lograr un uso óptimo de este árido como futuro agregado fundamental en la preparación de morteros de albañilería.

El tema del reciclado está referido en la literatura técnica y es tan antiguo como la construcción. La propia ciudad de Roma en la antigüedad, Londres, Berlín y
Varsovia, después de la II Guerra Mundial, son ejemplos de la reconstrucción a partir de sus propios escombros.

En Holanda y Bélgica se está llevando a cabo un programa conjunto de investigaciones sobre el reciclaje de escombros. También, en Francia, Japón, Alemaniay Gran Bretaña se estudia el tema. En toda Europa, salvo en España, los escombros de demoliciones de edificios y autopistas se reciclan; por ejemplo, en Alemania, se recicla un $60 \%$ de los escombros y en Holanda es superior al $80 \%$. En España existe, hasta la fecha, sólo una planta de reciclaje y en el resto de Europa alrededor de 150. En los Países Bajos se aprovecha un 73 \%. En los Estados Unidos existían, en 1981, 14 plantas para el reciclaje de los pavimentos de hormigón hidráulico, en cambio, en América Latina, ciudades que han sufrido desastres sísmicos, no han reciclado sus escombros $(1,2)$.

Con esos materiales reciclados se logran ventajas económicas, ya que se obtiene una fuente natural de 
materia prima, se reducen los gastos inversionistas de materiales, se sustituye un volumen de arena de mar, arena lavada o de árido integral para bloques, ahorrándose, además, por cada $\mathrm{m}^{3}$ de escombros que se desecha, 1 litro de petróleo, puesto que la distancia promedio a recorrer es de $14 \mathrm{~km}$ (3), o sea, se obtendrá, también con ello, ventajas energéticas y sobre el transporte, ya que, con sólo utilizar el $80 \%$ de los escombros de Ciudad Habana se ahorrarían $735 \mathrm{t}$ de combustible y se lograrían $91.500 \mathrm{~m}^{3}$ de árido reciclado $\mathrm{y}$, sobre todo, implicaría un gran impacto social, porque con ese árido es posible la fabricación de bloques para 4.000 viviendas (4), a la vez de ser un material alternativo que puede dar respuesta a la escasez de materiales, disminuyendo una de las principales fuentes de insalubridad, contaminación y deterioro ambiental.

Con ese volumen de materiales se puede apoyar una respuesta habitacional que incluya acciones constructivas, con recursos provenientes del reciclaje de los escombros, productos de derrumbes o demoliciones y su aprovechamiento en la creación de diferentes elementos constructivos, como el árido, para la producción de morteros de albañilería.

\section{MATERIALES Y MÉTODOS EMPLEADOS}

\subsection{Materiales}

\section{Cementos}

Se utilizó cemento Portland P-350, con las características que aparecen en las Tablas 1 y 2 .

\section{Agregados}

Comoagregado se empleó arena, producto de los escombros de la demolición de una edificación de Sistema Constructivo de Viga y Losa, de la década de los años 30 . No fue necesaria su trituración dadas las condiciones de los escombros, lo que permitió su empleo como arena, adaptándose con facilidad a las necesidades del estudio.

La muestra empleada fue homogeneizada y puesta en estufa, comprobándose que no contenia materiales orgánicos, virutas de madera, acero oxidado, fragmentos de cerámica, cristales u otrotipo de contaminante. También se realizó un análisis granulométrico.

\section{Agua}

Agua potable.

\subsection{Dosificaciones}

Se emplearon 4 dosificaciones volumétricas en los morteros de albañilería, las cuales son:

\begin{tabular}{|c|c|}
\hline $\mathbf{C}$ & $\mathbf{A}$ \\
\hline 1 & 4 \\
1 & 5 \\
1 & 6 \\
1 & 8 \\
\hline
\end{tabular}

\subsection{Métodos}

Los morteros de albañilería se caracterizaron empleando los siguientes métodos de ensayo:

\section{Ensayos fisico-mecánicos}

Probetas de $40 \times 40 \times 160 \mathrm{~mm}$ de morteros de albañilería fueron preparadas empleando la metodología que aparece en la NC52-79:93 y puestas en cámara húmeda con más del $90 \%$ de humedad relativa, hasta la edad de ensayo.

Los ensayos físico-mecánicos a flexión y compresión se realizaron de acuerdo con la NC54-207:80.

\section{Ensayo de absorción capilar}

Las probetas preparadas, según NC52-79:93, fueron puestas en la sala de ensayos, a temperatura ambiente, durante 6 dias y luego colocadas en posición vertical, sobre un lecho de arena fina, y sumergidas en agua hasta una altura de aproximadamente $3 \mathrm{~mm}$ (6). Después, se pesaron a las edades de ensayo, calculando la absorción en $\mathrm{g} / \mathrm{cm}^{2}$ de área sumergida.

\section{Ensayo de adherencia}

Los morteros fueron preparados de acuerdo a la NC5279:93 y adheridos a bloques de hormigón.

El espesor del morteros fue de $10 \pm 1 \mathrm{~mm}$. El ensayo se realizó de acuerdo al método del ensayo de adherencia descrito en el artículo de referencia (7).

\section{RESULTADOS Y ANÁLISIS}

En las Tablas 1 y 2 aparecen los análisis químicos, físicos y físico-mecánicos del cemento.

\subsection{Análisis de los resultados}

Los ensayos de cemento, realizados según la norma vigente NC54-205:87, muestran que el mismo cumple con las especificaciones de calidad.

El "ARESCO", o sea, el árido reciclado empleado como arena, se encuentra entre el rango de especificaciones contempladas en la norma NC54-125:78 (8) (Tabla 3). 
TABLA 1

Análisis químico del cemento

\begin{tabular}{|c|c|c|}
\hline PARÁMETRO & $\%$ & ESP. NC54-205:87 \\
\hline $\mathrm{SiO}_{2}$ & 21,01 & \\
\hline $\mathrm{Al}_{2} \mathrm{O}_{3}$ & 4,05 & \\
\hline $\mathrm{Fe}_{2} \mathrm{O}_{3}$ & 3,92 & \\
\hline $\mathrm{CaO}$ & 63,40 & $\%$ máx. 3,0 \\
\hline $\mathrm{MgO}$ & 2,81 & $\%$ máx. 3,5 \\
\hline $\mathrm{SO}_{3}$ & 2,44 & $\%$ máx. 3,0 \\
\hline $\mathrm{PPI}$ & 2,35 & $\%$ máx. 3,0 \\
\hline $\mathrm{RI}$ & 1,54 & \\
\hline
\end{tabular}

TABLA 2

Análisis físico-mecánicos del cemento

\begin{tabular}{|c|c|c|c|}
\hline PARÁMETRO & UM & P-350 & ESP. NC54-205:87 \\
\hline Consistencia normal & $\%$ & 24,4 & \\
\hline Fraguado inicial & $\min$. & 140 & $\min .45 \mathrm{~min}$. \\
\hline Fraguado final & $\mathrm{h}: \min$. & $3: 15$ & máx. $10 \mathrm{~h}$ \\
\hline Sup. especiffica & $\mathrm{cm}^{2} / \mathrm{g}$ & 3.533 & prom. 2.800 \\
\hline $\begin{array}{r}3 d \\
7 d \\
28 d \\
\end{array}$ & $\begin{array}{l}\mathrm{MPa} \\
\mathrm{MPa} \\
\mathrm{MPa}\end{array}$ & $\begin{array}{l}3,92 \\
6,51 \\
9,19 \\
\end{array}$ & $\begin{array}{ll}\min . & 3,0 \\
\min . & 4,0 \\
\min . & 5,5 \\
\end{array}$ \\
\hline $\begin{array}{r}3 d \\
7 d \\
28 d\end{array}$ & $\begin{array}{l}\mathrm{MPa} \\
\mathrm{MPa} \\
\mathrm{MPa}\end{array}$ & $\begin{array}{l}19,40 \\
28,80 \\
38,60\end{array}$ & $\begin{array}{cc}\min . & 17,0 \\
\min . & 25,0 \\
\min . & 35,0\end{array}$ \\
\hline
\end{tabular}

TABLA 3

Análisis granulométrico del árido

\begin{tabular}{|c|c|c|}
\hline TAMIZ $(\mathrm{mm})$ & ARESCO & ESP. NC54-125:78 (6) \\
\hline 4,76 & 92,2 & $90-100$ \\
\hline 2,38 & 82,4 & $80-100$ \\
\hline 1,19 & 61,8 & $50-85$ \\
\hline 0,59 & 35,8 & $25-60$ \\
\hline 0,295 & 15,0 & $10-30$ \\
\hline 0,149 & 7,0 & $2-10$ \\
\hline
\end{tabular}


Con respecto a los morteros de albañilería, solamente se encuentra normado, nacional e internacionalmente, el ensayo de resistencia a la compresión, intentándose lo mismo con el de adherencia. Para este estudio se tomaron las dosificaciones $1: 4 ; 1: 5 ; 1: 6$ y $1: 8$, donde el primer valor corresponde al aglomerante y el segundo al árido, en este caso, escombro reciclado. En todas las dosificaciones volumétricas, se utilizó la misma metodología de ensayos (Tabla 4).

En los resultados obtenidos en ensayos a compresión, la dosificación con mortero de escombros 1:8 es la que menor valor obtiene, $4,29 \mathrm{MPa}$, a los 28 días, valor que está por encima del mínimo exigido por la norma NC52-79:93, que es de 2,4 MPa, para morteros del tipo I, lo cual permite clasificar al resto de las dosificaciones dentro de los tipos II y III y, comparados con los morteros tradicionales (9), no es notable la diferencia, ni en esa dosificación, ni en las restantes.

La absorción capilar se determinó a las $8 \mathrm{~h}, 1,3,5$ y 7 días, obteniéndose valores muy parecidos a los resultados alcanzados en el trabajo de referencia (9) (Tabla 5). Se ensayó hasta los 7 dias, porque, a partir de esa edad, se mantiene prácticamente constante $\mathrm{y}$, además, para compararlo con los resultados de otros trabajos.
En el ensayo de adherencia, las mezclas diseñadas garantizan un buen agarre de los elementos de unión y de las terminaciones de los objetos de obra, donde pueden ser empleados, obteniéndose resultados comparables a los de referencia (9) (Tabla 6).

\section{CONCLUSIONES}

-El material proveniente de los escombros de demolición reciclado "ARESCO" es apropiado para la producción de morteros, ya que presenta un comportamiento similar a los áridos de cantera y de yacimientos.

-El empleo de los áridos reciclados de escombro produce un efecto económico por sustitución del uso de los áridos de cantera, tanto a nivel de industria como a nivel de obra, siendo mayor ese efecto en la medida en que se reduzca el transporte y el uso de energía en la producción de áridos y componentes.

-Efecto ecológico, por la eliminación de un desecho altamente contaminante (que es fuente de crecimiento de vectores antihigiénicos), por la reducción de los gastos de transporte, por la conservación de los recursos naturales (al permitir el empleo, más racional, de los áridos no renovables de las canteras), y, finalmente, por el efecto

TABLA 4

Análisis físico-mecánico de morteros de albañilería

\begin{tabular}{|c|c|c|c|c|c|c|}
\hline \multirow{2}{*}{$\begin{array}{c}\text { DOSIF. } \\
\text { VOL. }\end{array}$} & \multicolumn{3}{|c|}{ FLEXIÓN (MPa) } & \multicolumn{3}{|c|}{ COMPRESIÓN (MPa) } \\
\hline & $7 d$ & $28 d$ & $60 d$ & $7 d$ & $28 d$ & $60 d$ \\
\hline $1: 4$ & 2,60 & 2,63 & 3,06 & 4,47 & 11,11 & 14,51 \\
\hline $1: 5$ & 1.01 & 2,59 & 2,86 & 3,36 & 9,26 & 11,43 \\
\hline $1: 6$ & 0,92 & 1,84 & 2,04 & 2,73 & 6,69 & 9,14 \\
\hline $1: 8$ & 0,69 & 1,37 & 1,52 & 1,67 & 4,29 & 5,77 \\
\hline
\end{tabular}

TABLA 5

Absorción capilar $\left(\mathrm{g} / \mathrm{cm}^{2}\right)$ de morteros de albañilería

\begin{tabular}{||c|c|c|c|c|c|}
\hline $\begin{array}{c}\text { DOSIF. } \\
\text { VOL. }\end{array}$ & $8 \mathrm{~h}$ & $1 \mathrm{~d}$ & $3 \mathrm{~d}$ & $5 \mathrm{~d}$ & $7 \mathrm{~d}$ \\
\hline $1: 4$ & 1,25 & 1,98 & 2,26 & 2,33 & 2,46 \\
\hline $1: 5$ & 1,30 & 1,99 & 2,24 & 2,34 & 2,50 \\
\hline $1: 6$ & 1,25 & 1,97 & 2,28 & 2,35 & 2,49 \\
\hline $1: 8$ & 1,32 & 1,99 & 2,26 & 2,37 & 2,48 \\
\hline
\end{tabular}


TABLA 6

Ensayo de adherencia de morteros de albañilería

\begin{tabular}{|c|c|c|c|c|c|c|c|}
\hline \multirow{2}{*}{$\begin{array}{l}\text { DOSIF. } \\
\text { VOL. }\end{array}$} & \multicolumn{2}{|c|}{$\begin{array}{c}\text { FUERZA } \\
\text { APLICADA }\end{array}$} & \multirow{2}{*}{$\begin{array}{c}\mathrm{R} \\
(\mathrm{MPa})\end{array}$} & \multirow{2}{*}{$\begin{array}{c}\text { LUGAR } \\
\text { DE } \\
\text { ROTURA }\end{array}$} & \multicolumn{3}{|c|}{ RESULTADOS } \\
\hline & BAR & $\mathbf{N}$ & & & $x$ & MIN. & MAX. \\
\hline $1: 4$ & $\begin{array}{l}20 \\
20 \\
19 \\
24 \\
24 \\
16 \\
22\end{array}$ & $\begin{array}{l}1.550 \\
1.550 \\
1.500 \\
1.900 \\
1.900 \\
1.240 \\
1.640\end{array}$ & $\begin{array}{l}0,52 \\
0,52 \\
0,50 \\
0,63 \\
0,63 \\
0,41 \\
0,55\end{array}$ & $\begin{array}{c}\text { BM } \\
\text { BM } \\
\text { BM } \\
\text { BM } \\
\text { BM } \\
\text { R } \\
\text { BM }\end{array}$ & 0,54 & 0,41 & 0,63 \\
\hline $1: 5$ & $\begin{array}{l}20 \\
20 \\
24 \\
18 \\
20 \\
16 \\
20 \\
18 \\
\end{array}$ & $\begin{array}{l}1.550 \\
1.550 \\
1.900 \\
1.400 \\
1.550 \\
1.240 \\
1.550 \\
1.400 \\
\end{array}$ & $\begin{array}{l}0,52 \\
0,52 \\
0,63 \\
0,47 \\
0,52 \\
0,41 \\
0,52 \\
0,47\end{array}$ & $\begin{array}{c}M \\
M \\
M \\
M \\
B M \\
B M \\
M \\
M\end{array}$ & 0,51 & 0,41 & 0,63 \\
\hline $1: 6$ & $\begin{array}{l}12 \\
14 \\
14 \\
14 \\
14 \\
16 \\
16 \\
16 \\
\end{array}$ & $\begin{array}{r}900 \\
1.100 \\
1.100 \\
1.100 \\
1.100 \\
1.240 \\
1.240 \\
1.240 \\
\end{array}$ & $\begin{array}{l}0,30 \\
0,37 \\
0,37 \\
0,37 \\
0,37 \\
0,41 \\
0,41 \\
0,41 \\
\end{array}$ & $\begin{array}{c}\text { BM } \\
M \\
B M \\
M \\
M \\
M \\
M \\
B M\end{array}$ & 0,38 & 0,30 & 0,41 \\
\hline $1: 8$ & $\begin{array}{l}12 \\
13 \\
15 \\
14 \\
12 \\
13 \\
12 \\
12\end{array}$ & $\begin{array}{r}900 \\
1.000 \\
1.150 \\
1.100 \\
900 \\
1.000 \\
900 \\
900\end{array}$ & $\begin{array}{l}0,30 \\
0,33 \\
0,38 \\
0,37 \\
0,30 \\
0,33 \\
0,30 \\
0,30\end{array}$ & $\begin{array}{c}\text { BM } \\
\text { BM } \\
B M \\
M \\
B M \\
M \\
B M \\
B M\end{array}$ & 0,33 & 0,30 & 0,38 \\
\hline
\end{tabular}

NOMENCLATURA:

$\mathrm{BM}$ : Rotura unión bloque-mortero

R: Rotura resina

M: Rotura mortero

social, al contribuir al bienestar de la comunidad a través de la limpieza y el ornato público.

\section{RECOMENDACIONES}

Se recomienda su uso como mortero de albañilería del tipo I, II, III y IV, dependiendode la resistencia a la compresión a 28 días, según se expresa a continuación

\section{Tipo I: 2,4 MPa}

- Colocación de bloques de 0,15 y $0,20 \mathrm{~m}$ (interior).
- Colocación de ladrillo macizo o hueco de 0,15 y $0,20 \mathrm{~m}$ (interior).

- Colocación de losetas hidráulicas y baldosas de terrazo.

- Repello fino sobre resano interior.

\section{Tipo II: 3,5 MPa}

- Colocación de ladrillo macizo o hueco de 0,15 y $0,30 \mathrm{~m}$ (estructura exterior).

- Colocación de bloques de 0,15 y $0,20 \mathrm{~m}$ (exterior). 
-Resano y repello grueso.

-Repello fino sobre resano exterior.

*Tipo II, 5,2 MPa

- Colocación de losas de azotea.

- Colocación de rodapies y azulejos.
- Enchape de piedra Jaimanita sobre bloque o ladrillo.

*Tipo IV, 8,9 MPa

- Colocación de bloques de 0,065 y 0,10 m en tabiques.

- Enchape de piedra Jaimanita en superficie de hormigón.

- Colocación de ladrillos macizos o huecos de $0,10 \mathrm{~m}$ en tabiques o celosías.

\section{BIBLIOGRAFÍA}

(1) CASTELLS, E.: "Es preciso reciclar los residuos de demoliciones de las obras". Arte y Cemento, Madrid, 1994.

(2) FRONDISTOV, Y.: "Recycled concrete as new agregate in progress in concretetecnology". Canadian Gut. Public Centre. Ottawa, 1981. pp. 639-683.

(3) PADRÓN, J.: Director Empresa de Servicios Especiales de Edificaciones (Demoliciones). Comunicación verbal. febrero, 1996. Ciudad de la Habana.

(4) URRUTIA, F.: "Elaboración de materiales y elementos de bajo consumo para uso en la construcción, ARESCO". Ponencia. VII Forum. Ciudad de la Habana, 1992.

(5) NC52-79:93: Mortero de albañilería. Parte I. Diseño y caracterización. Código de práctica para la construcción. Vig. enero, 1993. $8 \mathrm{p}$.

(6) VENUAT, M.: “Absorción capilar. Aditivos y tratamientos de morteros y hormigones”. Barcelona, 1972. p. 66.

(7) ÁLVAREZ, J.L.: "La adherencia en los morteros de albañilería”. Materiales de Construcción. Vol. 45, No. 240. España, 1995. p. $47-52$

(8) NC54-125:78: Áridos para hormigón. Especificaciones de calidad. Vig. marzo, 1979. 5 p.

(9) ÁLVAREZ, J.L.: "Morteros de Albañilería”. Informe CTDMC. Ciudad de la Habana, 1995. 